\title{
Food for thought: Dietary influence on acetylcholine
}

\author{
Thomas B. Shea and Amy Chan \\ Center for Cellular Neurobiology and Neurodegeneration Research, University of Massachusetss, Lowell, MA \\ 01854, USA
}

We read with interest the excellent commentary provided by Dr. Lahiri. The role of methylation, as underscored in this commentary, has received further support by our recent study (in press in the Journal of Nutrition in Health and Aging [1], that demonstrates directly that SAM supplementation increases acetylcholine production. In this forthcoming report, we demonstrate that supplementation with SAM restricts the passive efflux of choline from brain tissue by methylating nicotinamide. The resultant increase in choline is then paralleled by increased acetylcholine. This represents an additional important function for SAM. Along with the demonstration by Scarpa and coworkers that SAM can prevent over-expression of presenilin-1 [2,3], and our prior demonstration that SAM is an obligate cofactor for glutathione-mediated quenching of oxidative species in brain tissue [4], these findings point towards SAM as a potentially critical nutrient in brain health. Notably, dietary supplementation with SAM compensated for dietary folate deficiency in all of these metabolic "hot spots," suggesting that consumption of SAM-rich foods, or at least consumption SAM in nutriceutical form (available over-the-counter) may be an important facet of nutrition, especially under conditions where folate availability may be limited by genetic deficiency in methylene terahydrofolate reductase (MTHFR) activity [5].

Lahiri raises the important question as to whether the neuroprotection provided by apple juice concentrate is a transient one that accompanies aging. Our studies using aged normal and ApoE-/- mice lead us to con- sider that genetic deficiencies can remain as latent risk factors that manifest only following critical nutritional compromise - and such compromise unfortunately is common in aging. The mechanistic model as presented by Lahiri nicely integrates the interaction of environment (nutrition) and gene in the context of development and aging, which is consistent with our studies.

In this regard, a final thought-provoking concern, presented by Reyes-Engel and coworkers [5], is that a $36 \%$ increase in C677T and A1298C polymorphisms of MTHFR has recently been reported among young people; such polymorphisms were present in $4.63 \%$ of individuals $>24 \mathrm{yr}$ of age, yet in $6.31 \%$ of those $<24 \mathrm{yr}$ of age. They considered that increased maternal dietary folate (confirmed in their samples) allowed an increase in fetal viability despite latent deficiencies in MTHFR; an increased latency within the population of critical genetic deficiencies in folate metabolism may therefore manifest only with age-related nutritional decline. Thus, as considered in this commentary by Lahiri, we may indeed be faced with an increased incidence of Alzheimer's disease unless the positive impact of a complete and balanced diet is brought to the attention of the public.

\section{References}

[1] A. Chan, F. Tchantchou, V. Graves, R. Rozen and T.B. Shea, Dietary and genetic compromise in folate availability reduces acetylcholine and cognitive performance: critical role of Sadenosylmethionine, J Health Nutr Aging (2006), in press. 
[2] A. Fuso, L. Seminara, R.A. Cavallaro, F. D'Anselmi and S. Scarpa, S- adenosylmethionine/ homocysteine cycle alterations modify DNA methylation status with consequent deregulation of PS1 and BACE and beta-amyloid production, Mol Cell Neurosci 28 (2005), 195-204.

[3] S. Scarpa, A. Fuso, F. D'Anselmi and R.A. Cavallaro, Presenilin 1 gene silencing by S-adenosylmethionine, FEBS Lett 541 (2003), 145-148.

[4] F. Tchantchou, M. Graves, D. Ortiz and T.B. Shea, S-adenosyl methionine: a link between nutritional and genetic risk factors in Alzheimer's disease, J Nutri Health Aging (2006), in press.

[5] A. Reyes-Engel, E. Munoz, M.J. Gaitan, E. Fabre, M. Gallo, J.L. Dieguez, M. Ruiz and M. Morell, Implications on human fertility of the $677 \mathrm{C} \rightarrow \mathrm{T}$ and $1298 \mathrm{~A} \rightarrow \mathrm{C}$ polymorphisms of the MTHFR gene: consequences of a possible genetic selection, Mol Hum Reprod 8 (2002), 952-957. 